\section{THE SURGICAL TREATMENT OF TYPHOID} FEVER.

By T. J. MACLAGAN, M.D., London.

ONE of the notable features of modern practice is the frequency with which the physician calls in the aid of the surgeon. In no department of medicine is this so marked as in the treatment of diseases of the abdomen. Liver, stomach, bowel, and kidneys are handled by the surgeon with a freedom and an impunity which our fathers would not have dreamed of as possible; and by aid thus given many lives are saved which without it would be lost.

If there is any class of diseases which the physician may regard as peculiarly his own it is the specific fevers. But even this domain has been invaded by the surgeon, and his help asked for in one of the most common of these feverstyphoid. It is the abdominal complications of that disease which give occasion for the surgeon's aid; it is, therefore, as a disease of the abdomen rather than as a fever that we have to regard it when we talk of its surgical aspects.

\section{Perforation.}

The most formidable complication of typhoid fever is perforation of the bowel. So formidable is it that it has been generally regarded as essentially fatal ; and so it used to be until recent advances in surgery, consequent on Lister's antiseptic system, suggested the idea of surgical aid.

some cases there are in which nothing could avail; the perforation is a large ragged opening (there may even be more than one), which allows of the free passage into the peritoneal cavity of the contents of the bowel. These cases would be more fittingly described as sloughing of all the coats of the bowel rather than cases of perforation. The opening is too large to be called a perforation ; it is not a perforation, it is a big hole, too big to be dealt with by the surgeon, and the patient too collapsed. for surgical treatment. Such cases are hopeless, and nothing can be done by either physician or surgeon.

Other cases there are, however, of a less severe character, with the symptoms of typhoid depression less marked, if not almost absent, in which true perforation occurs. In such cases there is generally only one opening in the gut, and that not a large one. There is not a very free escape into the peritoneal cavity of the contents of the bowel. The opening is not too large to be dealt with by the surgeon; and the patient's state not such as to forbid operation. These are the cases which may be saved by the surgeon, and which without his aid must die. But there must be no delay. All depends on early diagnosis of the accident, and prompt surgical aid before there is time for the development of general peritonitis.

How is the diagnosis to be made? Naturally one would say pain, pain at the seat of the perforation, would be the earliest symptom, and so it generally is, but not always. For be it borne in mind that some of these patients lie in a state of typhoid depression with more or less muttering delirium, and are not apt to give the usual indications by the sudden onset of pain, their state of blunted sensibility preventing them from feeling pain as they would otherwise do. Next to pain (in some cases before it) is to be placed altered character of the respiration. With the occurrence of perforation the respiratory act becomes shorter and more frequent, and is almost entirely thoracic; abdominal respiration is much diminished, and the abdominal walls, instead of being soft and mobile, are hard and rigid, and more so on the right than the left side. There is increased frequency of the pulse, a general appearance of distress, and may be hiccough and vomiting. But the three chief indications are pain in the lower part of the abdomen, diminished abdominal respiration, and rigidity of the abdominal walls. Any one of these suddenly showing itself towards the end of the third week of the disease points to perforation, their combination makes it a certainty, and the aid of the surgeon should at once be asked. Should there be a doubt, the patient should have the benefit of it. For even if it should prove unnecessary, the conse- quences of the operation are not serious; if there is perforation the consequences of leaving things alone are disastrous.

Cases in which hæmorrhage has occurred should be specially watched. Hæmorrhage means that the ulcer has eaten its way well into the muscular coat; it may eat its way through it, and penetrate the peritoneal

Opening the peritoneal cavity in cases of perforation of the bowel in typhoid fever is an operation which has been performed often enough, and with sufficient success to give it. the standing of a justifiable and proper procedure. In no case of suspected perforation should it be neglected except for special reasons. Dr. Osler, a strong advocate of early operation, states ${ }^{1}$ that in 11 of his cases operated on there were 5 recoveries, and gives it as his opinion that of cases thus treated, 30 to 40 per cent. may be saved.

Retained Sloughs.

Hitherto the aid of the surgeon in cases of typhoid has been limited to cases of perforation. But there is another. class of cases in which his aid might be called in with as much. possibly with greater, benefit.

In most cases of typhoid the sloughs and fœtid discharges which pass from the small into the large intestine are carried along the large gut without much delay, and are thrown off by the bowel with the diarrhœa which characterises the disease. But in a certain number of cases these sloughs and decomposing discharges are unduly retained in the large bowel, especially in the cæcum, where they undergo decomposition and fermentation, giving off gases which greatly distend the colon, and sometimes by over-distension produce a temporary paralysis of its muscular walls; the same sort of paralysis with which we are familiar in the case of an overdistended bladder-paralysis from over-distension.

The condition of the patient under these circumstances is as follows: He lies in a state of typhoid depression, there is muttering delirium or much restlessness; he takes no notice of what is going on around him; the tongue is dry or very dirty, the pulse feeble and rapid, the temperature $103^{\circ}$ to $104^{\circ}$ or even higher; food is taken with difficulty, or it may even be rejected; the abdomen is much distended, the contents. of the bowel may ooze away per anum as small, liquid, very offensive stools, generally of a dark colour. The condition is one of extreme and urgent danger; the patient is dying, not from the action of the typhoid bacillus, but from septic. poisoning, and from the pressure on the diaphragm of a much-distended colon. This latter it is which is the source of immediate and urgent danger, and it is an. hourly-increasing danger, for the continued presence and continued decomposition of the fotid bowel contents leads to greater and greater distension till the action of the diaphragm is interfered with, respiration impeded, and the cardias. action seriously embarrassed; and all this time the system is. being more and more poisoned and the septic condition becoming more and more pronounced. It is a hopeless state of matters and the sufferer must die, and die speedily, if something is not done to relieve him. Can anything be done? Occasionally the passage of a long tube gives relief by getting rid of some of the gaseous and liquid contents of the distended bowel, and so relieving the diaphragm. ln most cases such relief is only partial and temporary. It fails because the tube cannot reach the part of the large bowel in which the putrid and fermenting contents most abound, thecæcum and ascending colon, especially the cæcum.

The sole agency by which the contents of the large bowel are carried along to the rectum is the contraction of its muscular walls; this agency is put out of action by the overdistension of these walls. Some of the fluid contents of the gut may flow along and ooze away per anum, but the mass remains, the cæcum becomes a filthy cesspool, the system gets more and more poisoned by the absorption of septic material, and the diaphragm more and more pressed upon by the distended colon. Can nothing more be done?

On thinking over the matter some time ago, after death had in this way closed a valuable young life and a promising career, it occurred to me that in these hopeless cases it would be a right and justifiable procedure to open the cæcum. Consider what the state of the matter is. The cæcum and large bowel contain a mass of putrid and decomposing sloughs and discharges. The normal peristaltic action of the:bowels is in 
abeyance, and there is no natural means of getting rid of what is killing the patient--the retention in the large bowel of its decomposing contents. The best, I had almost said the only, chance of saving the patient is to open his cæcum and give exit to the poison which is killing him.

It is as a plea for surgical aid to such cases that this paper is written.

On looking back on the past I can recall several fatal cases in which I think the patient might have had another chance given to him by the aid of the surgeon. For what is the condition of such cases and what the cause of danger? It is no direct action of the bacillus, no essential outcome of the action of that poison which is killing the patient. It is late in the attack: he is completing his third week or has entered on the fourth; the sloughs have already separated or are coming away, and sloughs and fœtid discharges are passing from the small into the large gut; in most cases they pass on, causing more or less diarrhœa, which, though it may try the patient, has the undoubted advantage of carrying away the sloughing and putrid discharges; but in some cases they do not pass freely away-they lodge in the large bowel, especially in the cæcum; there they undergo fermentation and decomposition, giving off gases which cause more and more distension of the large bowel as they continue to be given off in increasing quantity. The results of this retention in the bowel of these fotid and decomposing contents are twofold : (1) poisoning of the system by the absorption of septic material ; and (2) great and embarrassing distension of the large bowel; either result may cause death; the combined action of both is almost essentially fatal. The only way to save the patient is to get rid of what is killing him-the retention in the cæcum and large bowel of its putrid contents. The only effectual way to do this is to open the cæcum. By so doing we should at once evacuate the colon of its gaseous and putrid contents, relieve the diaphragm, and arrest the process of septic poisoning. It is a bold step, but the condition is desperate, and boldness is called for. Since the idea of thus treating these cases presented itself to me I have had no opportunity to put it in practice. Did occasion arise I should not hesitate to recommend it. Without such relief the patient must almost certainly die, with it he might be saved. I make the suggestion in the hope that some of our hospital physicians who see most of such cases may share my views, and give to these desperate cases the chance which opening the cæcum would offer.

The operation would not be much; not nearly so great as that for perforation ; the opening would be a small one ; and no more than local anæsthesia would be called for. The result would be great, for the poisonous contents of the cæcum would find direct and immediate exit, and the danger of septic poisoning would be greatly diminished. The large bowel could be washed out, and discharges coming from the ileum got rid of as soon as they came down; the distended colon would return to its normal size, and the main obstacles to recovery be thus removed.

Here, as in the case of perforation, I should urge no unnecessary delay. Do not wait until the patient is in extremis, but so soon as there is evidence of interference with the action of the diaphragm, and the long tube fails to give relief, get a surgeon to open the cæcum.

As in the case of perforation, the risk of leaving things alone is enormous, the risk attendant on the operation almost nil. Let the patient have the benefit of the doubt and the safeguard of the operation. The chances against the patient if nothing is done are enormous. I am probably within the mark in saying that 90 or even 95 per cent. of such cases die. That is a mortality which justifies us in doing anything that gives a chance of recovery. And, after all, opening the cæcum is a less formidable procedure than opening the peritoneal cavity, which we do not hesititate to do in some cases of perforation.

The important point to be borne in mind is that what we have to deal with in these cases is not so much typhoid fever as septic poisoning; that it is not the ulcerated small intestine with which the surgeon has to deal, but over-distension of the large intestine consequent on the retention in it of sloughs and putrid discharges, which have passed from the ileum into the cæcum. In perforation the surgeon has to operate on a seriously damaged and diseased small intestine; in these septic cases he would be dealing only with a distended but. undamaged cæcum.

1 Lancet, REFERENCE.$$
\text { REFERENCE. }
$$

\section{A CASE OF TYPHOID ARTHRITIS PROPER.}

\author{
By JOHN J. BUCHAN, M.B., Ch.B.
}

Physician, County of Lanark Middle Ward Hospital, Motherwell.

CASES of enteric fever in which joint complications arise are very infrequent, and I have therefore thought it well to record the following case.

T. M., male, aged Ir, was admitted into Middle Ward Hospital, Motherwell, on February 5 th, rooo, notified to be suffering from enteric fever.

History. - Except for slight infantile troubles, patient had always enjoyed good health. His present illness began on January 20 th with headache and a feeling of cold and weakness. He complained of indefinite pains over the body, but these disappeared in a few days. He was restless anc sleepless at nights. His tongue became heavily coated, but he had not suffered from diarrhœea. On January 27th he complained of pain in his right elbow-joint, this was increased on pressure and movement. By February 4th $^{\text {th }}$ the pain in this joint had passed off, but on February 5 th the left elbow-joint became painful.

Condition on Admission. - On February 5 th, the sixteenth day of illness, the temperature was roc ${ }^{\circ}$. at I P,M., the patient was dull and sleepy, the pupils were dilated, and the conjunctivæ pearly and injected. The $a b-$ domen was somewhat distended, but there was neither tenderness no gurgling on pressure over it. Four typical rose-red spots were seen. The spleen was not palpable, but on percussion it came forward to the anterio axillary line. The tongue was fairly moist and covered with a thick white coat The heart was normal on examination, but in the lung occasional sonorous and sibilant rhonchi were heard. The urine contained no albumen, but presented the other ordinary febrile characters. Widal's test gave a positive reaction on the eighteenth day of the disease.

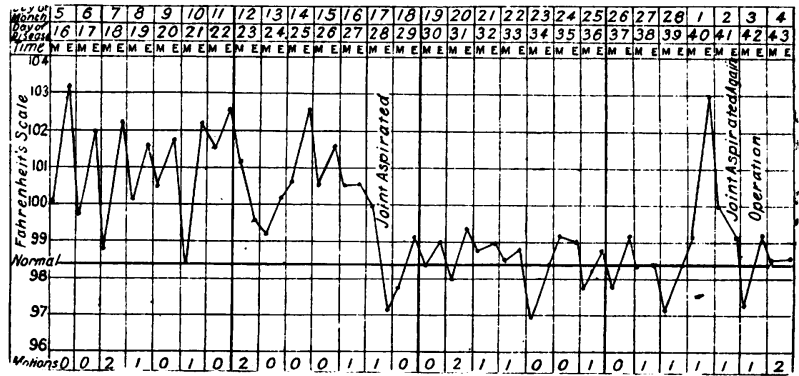

Progress.-While on admission the patient complained of pain, in creased on pressure and movement, in the left elbow-joint, no distinct preas of tendemess over the joint could be made out, and, in particular pressure over the ends of the bones did not reveal any tender spots. The pain complained of was not at all severe, and no swelling was noticed but on February gth, the pain continuing, the joint became dis tinctly swollen. At this time the forearm was pronated and flexed upon the upper arm. The joint was wrapped up in flannel for the first few days, but it was afterwards immobilised. On February 17 th the swelling of the joint seemed to be increasing, and it was resolved to aspirate the fluid; one drachm and a-half of clear serous fluid was drawn off which on bacteriological examination proved sterile. The aspiration was carried out with strict attention to the details of asepsis. The temperature fell to normal, and for a day or two the joint was not complained of. On February $24 \mathrm{th}$, however, pain was again felt and the swelling reappeared. On March rst the evening bemperature rose to 103 F., with, however, no distinct aggravation of the joint symptoms. On March 2nd aspiration was again resorted to, but this time a puru 等 number of streptococci and a lesser number of staphylococci. Carefu search was made for the bacillus typhor

With the help of Dr. Wyper I freely incised the With the help of Dr. Wyper 1 freely incised the joint and washed it out in every part with $r$ in 8 carbolic lotion. During this process the daily for the first few ded an found intact. The wound was dressed the time the patient was discharged on May r6th, movement had been the time the patient was

Very little notice has been taken of the joint complications of enteric fever. The literature of the subject consists almost wholly of isolated cases appearing from time to time in the medical journals. Keen ${ }^{1}$ has recently collected 84 such cases, and has divided them into three classes: rheumatic typhoid arthritis, septic typhoid arthritis, and typhoid arthritis proper. To these classes Dreschfeld ${ }^{2}$ has added a fourth, osteoarthritis, where typhoid osteitis is situated near to and extending into a joint.

Taking these four classes into consideration, the diagnosis in the present case would seem to rest between rheumatic 